\title{
Some remarks on climatic maps of precipitation
}

\author{
P. A. Kay ${ }^{1}$, H. Kutiel ${ }^{2}$ \\ ${ }^{1}$ Department of Environment and Resource Studies, University of Waterloo, Waterloo, Ontario, Canada N2L 3G1 \\ ${ }^{2}$ Department of Geography, University of Haifa, Haifa 31905, Israel
}

\begin{abstract}
Issues in the accuracy of preparing and using isoline maps of mean precipitation are reconsidered. A simulation is created to illustrate the concepts of discontinuous distribution and the effects of sampling on the resulting maps. The actual rainfall field is more closely represented as the number of rainfall events considered and the grid density increase. Data from Israel are used to provide concrete illustrations. A method of interpolating to a fine grid is proposed, using a regression model of station precipitation related to geographical variables of latitude, longitude, and elevation.
\end{abstract}

KEY WORDS: Climatic maps · Isohyets · Rainfall fields · Israel

\section{INTRODUCTION}

The spatial properties of precipitation, like most climatic variables, are often depicted by means of isoline maps. The preparation of isoline maps rests on 3 assumptions regarding the nature of the variable being mapped. First, the variable is assumed to be continuous in space, so that values may be interpolated between known values measured at a few places. Moreover, the spatial distribution (the field) is often thought to be isotropic, that is, that it changes in a smooth regular way between the known values. Second, the known values are assumed to be representative of some area around the point of measurement. The interpolation technique of Thiessen polygons (also known as Voronoi tesselations), for example, is indicative of this assumption. Third, the mean is often used as a summary statistic of the variable to represent, at least implicitly, the most commonly occurring value.

Sumner (1988) noted the drawbacks of isohyets, yet stated that they 'portray precipitation in a spatially continuous manner, which matches our perception of the reality of the distributions... When carefully used they will present a reasonably realistic picture' (p. 401-402). But is this perception of the continuous distribution of precipitation accurate? And, are there criteria for 'careful' application of the concept of isohyets? In this paper, we examine the assumptions in both a conceptual model and empirical evidence. We propose an alternative to isoline mapping of mean precipitation from station data that provides a more realistic picture of the precipitation field.

\section{CONCEPTUAL CONSIDERATIONS}

\section{Continuity}

If we consider a single precipitation event, it is clear that space can be differentiated into a region receiving precipitation and a region without precipitation. While there is a measurable amount of precipitation under the generating cloud, there is none beyond the edge of the cloud. Moreover, several studies show precipitation events may comprise several cells precipitating at various intensities, so it is not certain that within the event's edge the precipitation is distributed uniformly or isotropically (Sharon 1972, Berndtsson 1988, Jackson 1989, Linacre 1991). Two events that are spatially distinct will have a region of zero precipitation between them. Such events may be the cells within a mesoscale convective complex or along a front, or may be temporally distinct rainfalls. As it is limited by zero at its lower bound, precipitation cannot be considered as a spatially continuous variable. The property 'precipitation received in an event' thus possesses a zeroorder discontinuity (Hess 1959, p. 229-230). For variables such as temperature or pressure, one can be sure 
of the existence of an isotherm or isobar, once 2 values are measured, one above and the other below the isoline value (although the exact position or shape of the isoline may be in doubt). This certainty does not exist for precipitation, as there is no dynamic boundary condition requiring continuity across the boundary. The zero isohyet delimiting the precipitation event is a true discontinuity. Care must obviously be exercised in drawing isohyets in a region where or for times when isolated precipitation events occur.

A practical illustration of this discontinuity is seen in storm data for the Gulf of Eilat region (Israel and Jordan) first published by Sharon (1979). The distance between Eilat and Aqaba is about $5 \mathrm{~km}$ and between Aqaba and Wadi Yatoum about $10 \mathrm{~km}$ (Fig. 1). This density of stations is exceptional and infrequent in such an arid region. The variability of the measured rainfall is enormous; the ratio of largest to smallest amounts exceeded 20 in $50 \%$ of the events. We estimate isohyets for the area based on the 2 stations at either end of the transect (a distance of only $14 \mathrm{~km}$ ), assuming a linear change between the two. The rainfall that would be estimated for the middle station
(Aqaba) may vary very considerably from what was measured in reality (Fig. 1)

As we aggregate precipitation events in time, say to monthly, seasonal, or annual scales, we find a marked (we might almost say, a discontinuous) change in the spatial expression of precipitation. Because succeeding events usually do not coincide exactly in space, a spacefilling behavior occurs. With more and more events, all parts of a region are likely to receive some precipitation, so that the property 'total precipitation received' is not likely to possess zero-order discontinuity. Isohyets crossing the ungauged space between stations may therefore be generalized, with an implicit understanding that they represent a statement of probability that precipitation is received and is of the amount designated. The more rugged the terrain, the more varied the precipitationgenerating mechanisms and thus the precipitation field. The more dense the observing network, the more likely the isohyets will depict the complexity of the field. If precipitation is considered as a function of distance, the gradient of precipitation (i.e. its spatial derivative) may possess a first- or second-order discontinuity (Hess 1959, p. 229-230).
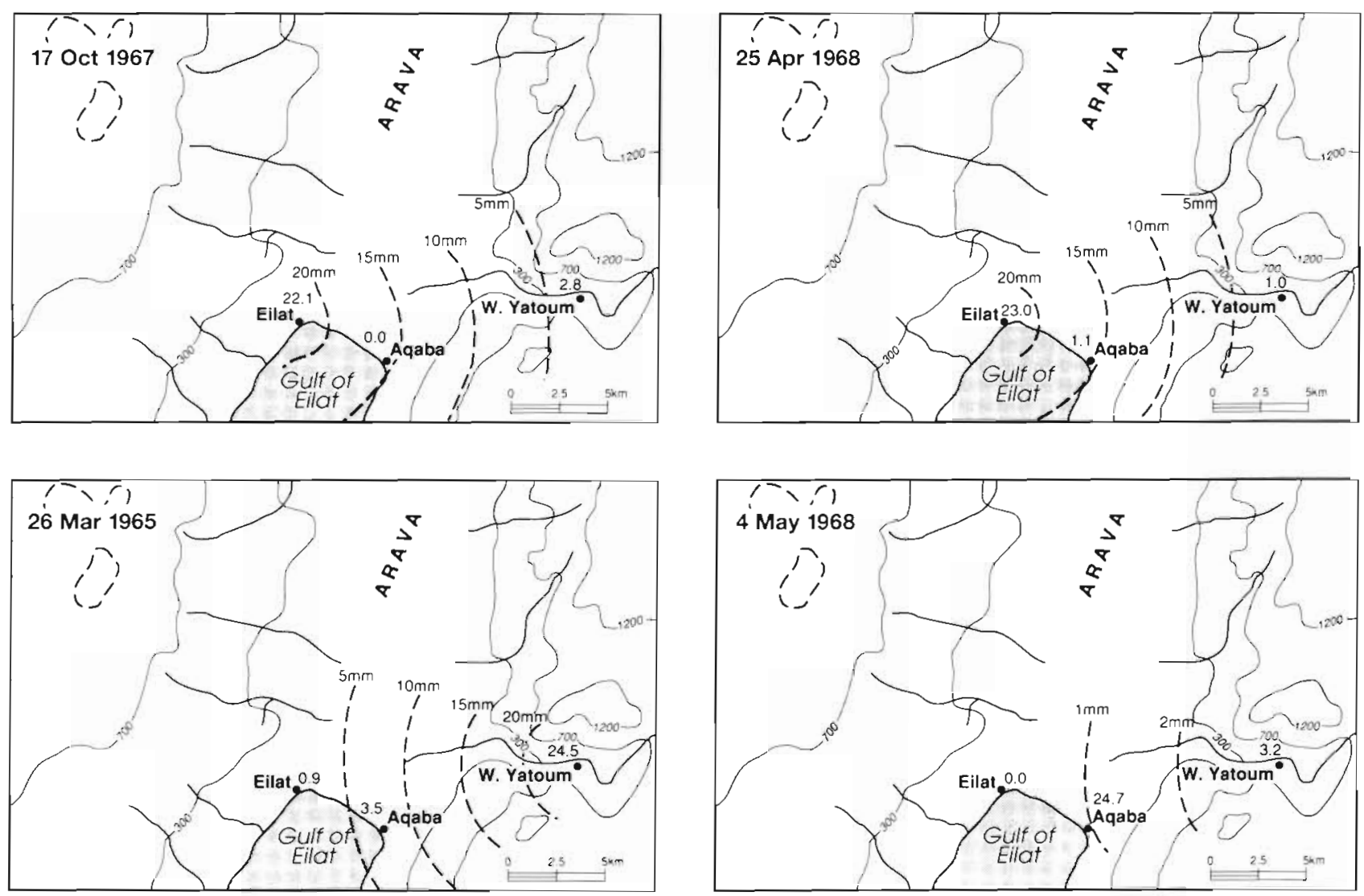

Fig. 1 Four ranfall events in the northern Gulf of Eilat area, Israel. The isohyets are based only on data from Eilat and Wadi Yatoum. Note the large interpolation errors at Aqaba, compared to the actual amounts received. (Rain totals after Sharon 1979) 
Fig. 2. Outlines (0-isohyets) of 2,5, and 10 'left to right, respectively) random elliptical events. The region of interest is shown by the square
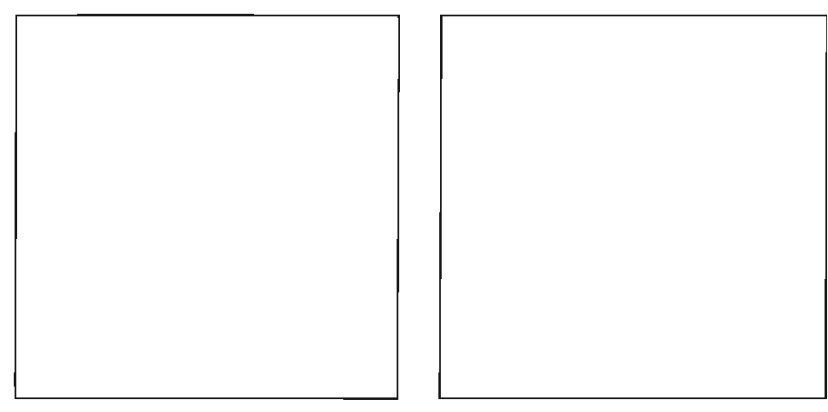

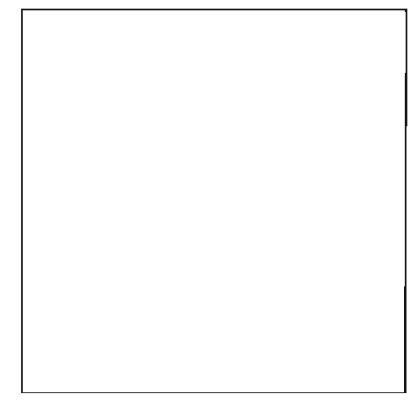

Where along the continuum from individual events to temporal aggregation does the zero-order discontinuity disappear? What effect does network density have on our appreciation of the behavior of the precipitation field? While we cannot give quantitatively specific answers, the following simulation is illustrative.

For the simulation, we assume that the precipitation field represents amalgamation of some number of distinct organized precipitation events. These events may be thought of as short-term events in a single mesoscale phenomenon, individual events in a month or season, or monthly or seasonal total experiences aggregated into the long term. Sumner (1988) noted that most precipitation events are elliptical, with the long axis oriented parallel to the direction of motion of the event. We therefore represent each precipitation event by a set of nested elliptical isohyets generated from the usual equation for the geometric figure:

$$
\frac{x^{2}}{a^{2}}+\frac{y^{2}}{b^{2}}=1
$$

For this simulation, we assume that the eccentricity of the elliptical events is a uniform random variate, and that the location of the centre of each event (relative to a fixed geographic origin) is a normal random variate. These elements of randomness represent in the simulation the variability due to precipitation-generating mechanisms, size of event, and other complexities of atmospheric organization and dynamics.

Each elliptical event was depicted as a set of nested elliptical isohyets representing $0.0,0.3,0.6$, and 0.9 of maximum precipitation amount located at the centre of the ellipse. The isohyets, therefore, gave fixed values for the vertices (a) of the ellipses on the major axis (set coincident with the $x$-axis). The foci (c) were then calculated from the eccentricity $(e=c / a)$, which was chosen at random from a uniform distribution on the range 0 to 1 . Corresponding values of the vertices (b) on the minor axis were then calculated (from $b^{2}=a^{2}-c^{2}$ ). The location of each elliptical event relative to a fixed geographical origin was determined by choosing random increments for both $x$ - and $y$-axes from the standard normal distribution. For this simulation, we assumed precipitation events of equal magnitude.

Using the zero isohyet to delimit the elliptical events, the space-filling phenomenon is seen to occur after only a few events (Fig. 2). Although 2 events may be spatially distinct, 10 random events sufficiently overlap to provide precipitation to most of the region. To estimate the total precipitation represented by the 10 events, we overlaid a regular rectangular grid and interpolated from each set of nested ellipses the point values of precipitation (Fig. 3). In statistics, the central limit theorem states that the mean of the sampling distribution of event means equals the mean of the population from which the samples were drawn (Newmark 1991). Our usual concept that the aggregated rain field consists of the sum of the individual events, and represents the mean experience or expectation of those events, is consistent with this theorem. Although for a finite number of rainfall

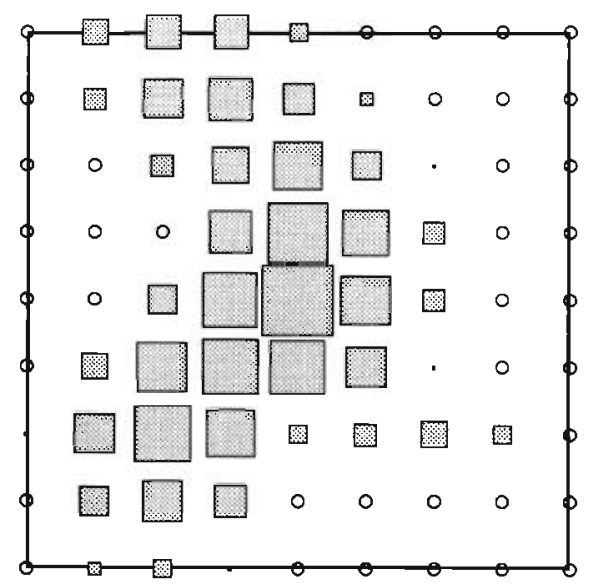

Fig. 3. Precipitation totals estimated on a regular rectangular grid from the 10 random elliptical events. The area of each shaded box is proportional to the rainfall total; open circles are grid points without precipitation 

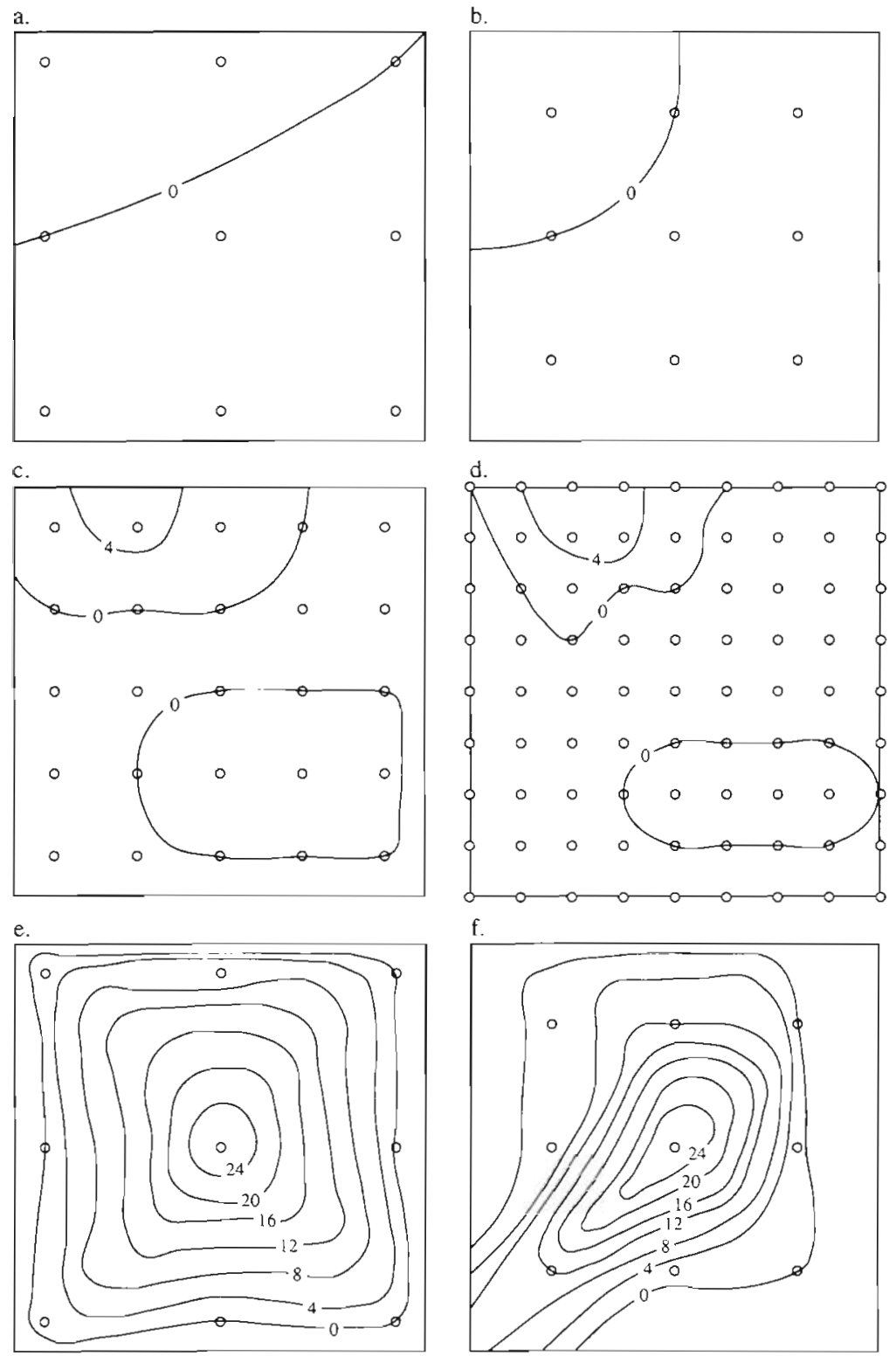

g.

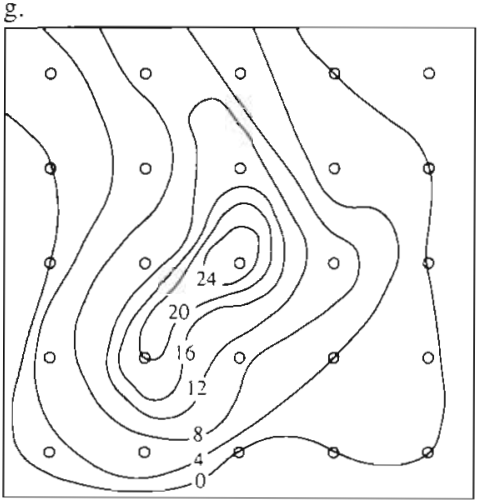

events we can never be certain that precipitation received at sites between gauges is continuously distributed between the values at the gauges, the statistical interpretation at least suggests that is so. That is, within the region when many events are aggregated into a total, precipitation does not possess a zero-order, and likely neither a firstorder, discontinuity. The finer the grid mesh used to sample rainfall, the more likely we are to test or evaluate the scale at which this interpretation is valid.

\section{Representation}

To illustrate the effect of network density, we sampled the aggregated field generated by the elliptical events with grids of varying mesh size. The choice of grid sizes was based on consideration of real-world networks, as follows.

Among the densest national networks are the United Kingdom and Israel, in which the number of gauges represent (if uniformly distributed, which is recognizably not the case) an area of approximately 50 to $60 \mathrm{~km}^{2}$ per gauge (Sumner 1988, p. 301). This density suggests a uniform rectangular spacing of about $7 \mathrm{~km}$ between gauges. Such a grid would seem to be sufficient to sample the complexity of most precipitation events. Many national networks, however, are 1 or more orders of magnitude less dense. For example, Stephenson's (1968) data suggests 25 gauges for an area the size of Israel and 35 for an area the size of the UK are adequate for monthly rainfall, and the WMO (1965) recommended 100 to $250 \mathrm{~km}^{2}$ per gauge for mountainous areas, and 600 and $900 \mathrm{~km}^{2}$ per gauge for flat areas, under normal tolerance. The phenomenon of non-uniform placement of stations is also well known (Sumner 1988).

Even at the densities of gauges in Israel and the United Kingdom, we may still question the representation by point data for areal estimation. For example, if the approximately 450 rain gauges in Israel are assumed to have a standard orifice of $200 \mathrm{~cm}^{2}$ (there are smaller
Fig. 4. Isohyets inferred for $2(\mathrm{a}-\mathrm{d})$ and $10(\mathrm{e}-\mathrm{h})$ random elliptical events from rectangular grids of spacing of 1 (upper left), 0.5 (upper right), 0.25 (lower left), and 0.10 (lower right) times the median size of the events 
gauges with orifices of only $8 \mathrm{~cm}^{2}$ ), the total sampled area would be $90000 \mathrm{~cm}^{2}$. The ratio between the sampled area and the area to be sampled $\left(27000 \mathrm{~km}^{2}\right)$ is $1: 3 \times 10^{9}$. Most other nations do not fare so well. For example, the equivalent figures for France, Tunisia and Algeria are $1: 8 \times 10^{9}, 1: 13 \times 10^{9}$, and $1.5000 \times$ $10^{9}$, respectively (Kutiel 1993). Clearly, then, finer grids should be desirable, depending on the application for which the rain field will be used.

We therefore chose grids representing stations spaced on a rectangular grid of $4,2,1,0.5,0.25$, and 0.1 times the median size of our elliptical events. Isohyets were interpolated between the point estimates of total precipitation, based on no 'knowledge' other than the precipitation amount and a rule of linear interpolation (Fig. 4). When the events are few in number, some may be missed entirely by the network, and inadequate sampling of others may lead to misspecification of magnitude and extent. When there are frequent events, the interpolation is likely to lead to a bulls-eye effect centred on the mean position of the spatial distribution. When the size of the grid is larger than the typical size of precipitation events, a smooth-appearing but misleading field emerges. Many events may 'slip through' the grid and go unsampled, and the resulting isohyetal map may be blank or consist of a single point estimate. As the grid becomes finer, in relation to the typical size of events, the interpolation better represents the structure and complexity of the accumulated precipitation.

The implications of the simulation are familiar. The more frequent rainfall events are in a region, the more likely the space-filling behavior is. The finer the network mesh, the better the interpolation captures the complexity of the rainfall experience. In fact, if the simulation even closely mirrors reality, it seems that space-filling occurs rather rapidly (that is, without too many events). The need for a dense, well-positioned grid persists, and efforts to enhance spatial representation may be well rewarded when the spatial characteristics of the precipitation are of critical concern (for example, in hydrological applications).

\section{Central tendency}

Although we have talked about and used the mean in the simulation above, it is worth reiterating some properties of the mean as a measure of central tendency of a distribution. The mean as a representation of a series of observed values is appropriate only if the variability of the observations around the mean is not large, if the distribution is symmetrical around the mean, and if the frequency of observations decreases as the distance from the mean increases. If these

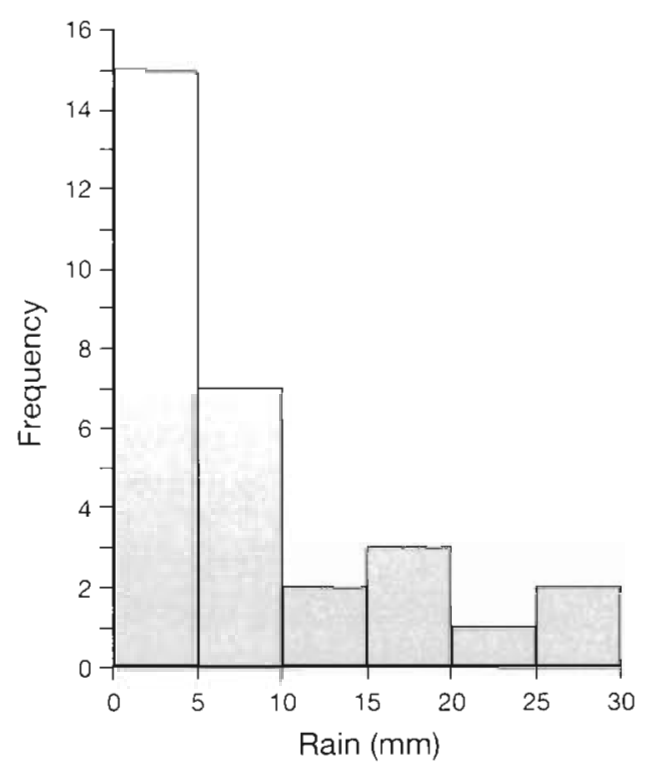

Fig. 5. The distribution of monthly rainfall in December at Eilat, Israel, during the years 1949 to 1978

assumptions do not hold, such as when the distribution is skewed, the mean is not located at the center of the distribution. These conditions are very often violated by precipitation data, particularly in arid and semi-arid regions. In these regions, the variability is very large the standard deviation of the data is of the same order of magnitude or even greater than the mean, and the distribution is very skewed (usually positively). For example, the distribution of December rainfall in Eilat, Israel, from 1949 to 1978 , is asymmetric and positively skewed (Fig. 5). The standard deviation was $8.2 \mathrm{~mm}$ $22 \%$ greater than the average of $6.7 \mathrm{~mm}$. In 19 of the 30 years, below-average rainfall was recorded, while above-average rainfall occurred in only 11 years. The ratio between below- and above-average occurrences was almost $2: 1$, whereas in a symmetric distribution we would expect a ratio of $1: 1$. In cases such as this, a year with average rainfall may in fact be considered an exceptional year. The representation of such data by the mean may therefore lead to confusion and inaccuracy regarding implications for both frequency and magnitude.

\section{OVERCOMING THE PITFALLS OF ISOLINE MAPS}

Isohyets are usually interpolated as rounded and simplified isolines, when in fact the actual spatial pattern of precipitation is much more complex. The stations providing the data are usually not evenly distributed over all the area shown by the map. The accuracy of the isolines and thus of the whole map is 
not therefore equal over all the area shown by the map. The data used for preparing the map are only a sample of the whole population of the variable. Samples usually do not include the highest and lowest values. However, the usual tendency of the map makers is not to exceed the measured range of values. The interpolation of the isolines is usually done in a 'technical' way, such as linear interpolation. Geographical or physiographical conditions of the area, which may impart important variation to the rainfall field, may be reflected qualitatively in the drawing of the isohyets, but the interpolation scheme is usually paramount.

These shortcomings could remain only theoretical, if the maps served to represent only the trends of spatial variability of the mapped variable. However, there is an extensive use of climatic maps for various purposes. For example, rainfall maps may be used to calculate the water volume that fell over a certain region (e.g. Stanhill \& Rappaport 1988). The calculation of water volumes from isohyetal maps when the concept of isohyets is erroneous, the area included between 2 adjacent isohyets is not accurate, and the average overestimates the rainfall regime, may lead to important errors of the water volumes. These facts may be crucial in a country like Israel, were water resources are fully utilized (Sadan \& Ben-Zvi 1987).

Bearing these sources of error in mind, 2 choices at the beginning of the analysis may help produce more accurate results.

\section{Choosing the mapped variable}

The median is a more robust measure than the mean of the central tendency of a non-symmetric distribution. The median is not sensitive to extreme values. The median is thus very useful to describe rainfall in arid and semi-arid areas, where most rainfall comes from local convective storms precipitating at high intensities (e.g. Sharon \& Kutiel 1986). One such storm measured at a single station may raise the average a great deal and change the inferred rainfall pattern in that region. The advantage of the median over the mean in more humid regions is reduced somewhat; however, even in humid regions the rainfall may not be normally distributed (e.g. Striem 1967). The use of the median introduces a probabilistic aspect into the map, because $50 \%$ of the observations are smaller and $50 \%$ of the observations are larger than the median. Although this probabilistic interpretation is implicit in the use and interpretation of the mean, it is increasingly misleading and incorrect as the mean differs from the median (as the frequency distribution is skewed)

\section{Choosing the mapping technique}

One way to overcome the shortcomings of mechanistic isohyet interpolation is to describe the spatial distribution of precipitation according to geographical variables. Latitude and longitude may capture the influence of synoptic controls, and elevation the orographic effect. Several previous studies in Israel fit multiple regression models to the spatial distribution of the rainfall (Rosenberg 1969, Diskin 1970, Wolfson 1975, Kutiel 1987, 1988, 1989). However, in none of these studies were rainfall maps prepared.

The proposed technique begins with a data base of precipitation, latitude, longitude and elevation, for as many stations as possible. Multiple regressions of precipitation with these geographic variables are calculated, and estimates of precipitation are derived for a fine mesh of grid points. The resulting map is much more complex and thus more realistic than the usual isohyet map. The problem of an uneven distribution of stations is overcome, as the maps are drawn from values calculated at evenly spaced grid points. The range of the mapped values is not restricted to the measured values, as the model allows for extrapolation based on the geographical values. The effect of geographical or physiographical conditions of the area on the variable are taken into account in a quantitative way.

\section{MAPPING RAINFALL OF ISRAEL}

To illustrate the advantages of the proposed mapping technique over maps prepared in the usual manner, several maps of rainfall in Israel were prepared Thirty-nine rainfall stations were selected from the network of the Israel Meteorological Service (IMS). The data pertain to the rainy seasons $1960 / 61$ to $1991 / 92$. In the few cases of missing data at a certain station, a value was estimated using a nearby station.

\section{Mapping average and median rainfall using isolines}

The mean precipitation field (Fig. 6a) is dominated by a gradient from $100 \mathrm{~mm}$ in the northern Negev (less at Eilat) to over $800 \mathrm{~mm}$ in the northern Galilee and the Golan. The field of median precipitation (Fig. 6b) is very similar, but close examination reveals a northward shift of the isohyets. With the exception of 1 station (Gaza), the medians are lower than the means (Fig. 6c). The greatest difference is in the center of the country around Jerusalem, where the median is lower than the average by 50 to $60 \mathrm{~mm}$. When the difference is expressed as a percentage of the mean, the largest 
discrepancies occur mainly in the arid Negev desert, where values exceed $10 \%$ (Fig. 6d)

The number of years in which average or below-average precipitation was measured exceeded $50 \%$ at all stations (except Gaza). At Jerusalem Airport, the value was $66 \%$. Thus, a year with an average amount of rain should be regarded as exceptional rather than as expected or the norm in most of Israel.

\section{Mapping rainfall calculated by a regression model}

The isoline map of mean precipitation (Fig. 6a), based on a sample of only 39 stations, compares favorably with published maps prepared by the Israel Meteorological Service based on 450 gauges (e.g. Israel Survey 1985). An 11 -fold increase in the number of stations used, from 39 to 450 , refines the map only slightly. Thus, for general purposes, one may use a relatively small sample comprising a few tens of gauges (carefully choosen) as suggested by Stephenson (1968). However, if a finer description of the rainfall distribution is needed, increasing the number of the gauges may not be as important as using a mapping technique that incorporates geographical information. Certainly in situations where only a coarse-grid network exists, it may be impractical to establish new gauges and wait for a sufficient number of years to obtain fine-grid data.

Several studies have previously used regression models (mainly with elevation) for interpolation purposes. Aleman \& Garcia (1974) used topographic contours to interpolate isotherms for Mexico. Pielke \& Mehring (1977) and Groisman \& Legates (1994) noted the inadequacy of most contouring schemes from station data and the need for inclusion of elevation in mountainous terrain, for both temperatures and

Fig. 6. Comparison of mean and median precipitation for the period $1960 / 61$ to $1991 / 92$. (a) Isolines of mean rainfall. (b) Isolines of median rainfall (box shows the area of Fig. 7). (c) The difference (mean-median). (d) The difference as a percentage of the mean
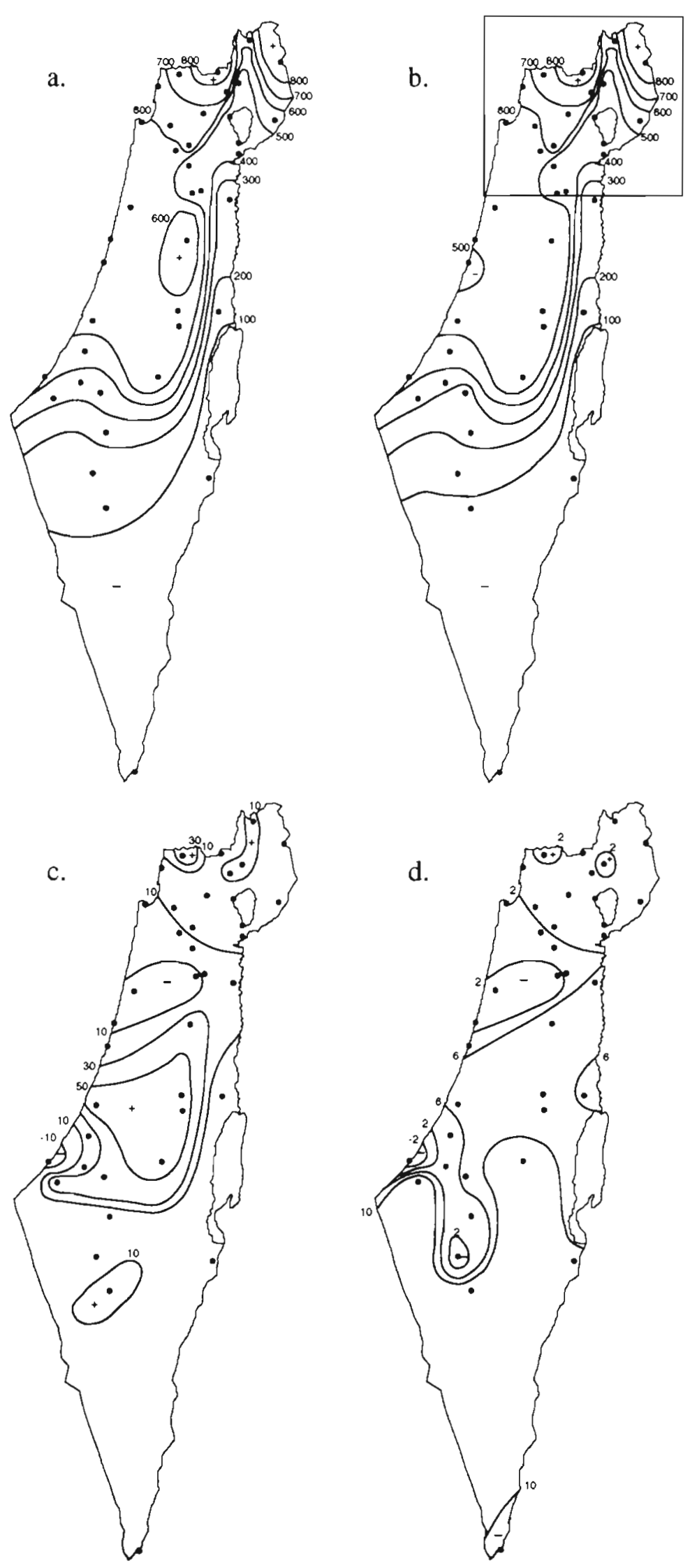


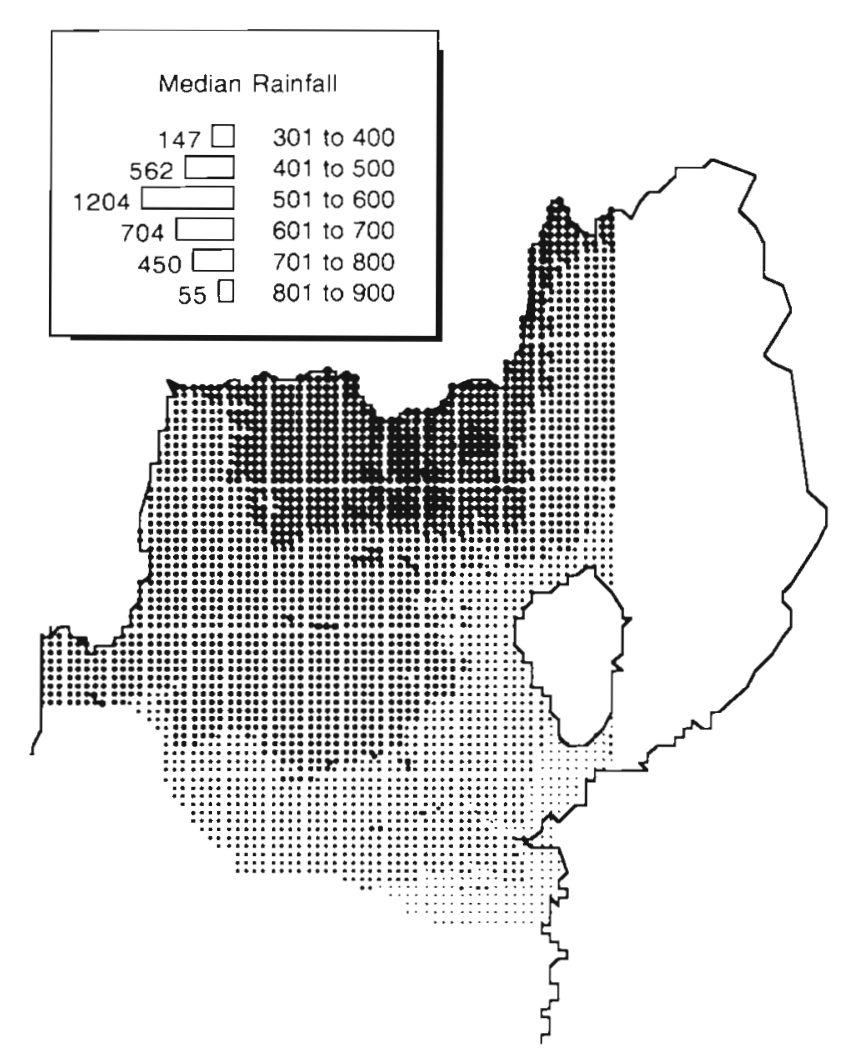

Fig. 7 Median rainfall $(\mathrm{mm})$ in the Galilee for the period $1960 / 61$ to 1991/92, calculated by the method of multiple regression on geographical factors. The histogram shows the number of data points in each rainfall class. Magnitude of rainfall is proportional to dot density

hydrological applications. Eyton (1986) used a regression model with elevation to map mean annual temperatures in Pennsylvania. Russo et al. (1993) modelled maximum and minimum temperatures from regression with longitude, latitude and elevation, in order to predict hatch dates of gypsy moth eggs.

The technique is illustrated by considering the Galilee region of northern Israel. The Galilee extends from $200 \mathrm{~m}$ below sea level (Lake Kinneret) to over $1200 \mathrm{~m}$ (Meron Mountains in the Upper Galilee), and is mostly hilly. The field of mean precipitation bears the imprints of distance from the Mediterranean (a source of moisture and heat), distance from the mean storm track that is aligned from Cyprus eastward across Syria, orographic effects, and windward and leeward effects. The geographical variables influencing the spatial distribution of the rainfall in the Galilee are, therefore, latitude, longitude, and elevation (Rosenberg 1969, Diskin 1970, Wolfson 1975, Kutiel 1987. $1988,1989)$. A multiple regression analysis between these 3 variables and the median rainfall at 18 stations gave the following model:

$$
\begin{aligned}
\text { RAIN }_{\text {median }}= & 66.8+3.038 \mathrm{LAT}-1.645 \mathrm{LON} \\
& +0.275 \mathrm{ELEV}
\end{aligned}
$$

where RAIN median $_{\text {is }}$ the median rainfall for the period $1960 / 61$ to $1991 / 92$, LAT and LON are respectively the latitude and the longitude of the stations according to the Israeli national grid, and ELEV is the altitude of the stations in meters above sea level. The coefficient of determination (explained variance) was 89.6\%, and the model was statistically significant at probability of 0.01 . All 3 coefficients were also significant at less than 0.05 . In order of contribution to the explained variance, elevation and latitude were most important.

A regular network of 3152 points on a $1 \mathrm{~km} \times 1 \mathrm{~km}$ grid was prepared. For each point of the network the 3 geographical variables were measured. The median rainfall at each grid point was then calculated from the model and mapped by a plotter using Atlas Graphic (Fig. 7). The isopleths are shown in steps of $100 \mathrm{~mm}$, corresponding to the usual isoline interval on the maps of Israel but the technique is readily generalizable to finer resolution. The map resembles the general isoline map (Fig. 6b) but is much more complex, a fact that makes the present map more realistic. The extreme values in the present map are out of the range of the measured values, again a fact that makes the present map more realistic.

\section{CONCLUSIONS}

We have demonstrated a new approach in mapping precipitation that deals with most of the shortcomings of isohyet maps. The technique can be applied to other climatic variables as well. In the present approach, isolines are not fit to the measured data, but rather those data are used to calculate the coefficients of a multiple regression model describing the spatial distribution of the mapped variable according to geographical controls. The coefficients are then used to calculate the desired variable on a dense network of grid points. These calculated values are then mapped. Another important point that was illustrated is the biased representation of central tendency given by the mean, and the preference for the median instead.

The biggest disadvantage of the proposed approach is the relatively enormous amount of work that preparation of the network of grid points entails. However, once this network is prepared, it may be used again and again for mapping different variables. It may also serve for the preparation of maps of a combination of several variables. The preparation of such a combination map in conventional ways is a tremendous work, compared to the ease in the present approach. The compatibility with the technology of geographical 
information systems (GIS) is readily apparent, and we may expect that improved climatic maps of single and combined variables will be produced and integrated with other environmental and social variables in GIS applications

Acknowledgements. The authors thank Ms Z. Gilboa of the Israel Meteorological Service for supplying the rainfall data and R. Haas, E. Zefadia, and D. Zvieli of the Department of Geography, University of Haifa, for help in preparing the maps.

\section{LITERATURE CITED}

Aleman, P. A. M., Garcia, E. (1974). The climate of Mexico. In: Bryson, R. A., Hare, F. K. (eds.) Climate of North America. World survey of climatology, Vol. 11. Elsevier, Amsterdam, p. $345-404$

Berndtsson, R. (1988). Spatial hydrological processes in a water resources planning perspective. Report No. 1009, Department of Water Resources Engineering, University of Lund

Diskin, M. H. (1970). Factors affecting variations of mean annual rainfall in Israel. Bull. int. Ass. scient. Hydrol 15 $41-49$

Eyton, R. J. (1986). Digital elevation model perspective plots overlays. Ann. Ass. Am. Geographers 76: 570-576

Groisman, P. Y., Legates, D. R. (1994). The accuracy of United States precipitation data. Bull. Am. Meteorol. Soc. 75: $215-222$

Hess, S. (1959). Introduction to theoretical meteorology. Krieger Publishing Company, Malabar, FL

Israel Survey (1985). Atlas of Israel. Tel-Aviv

Jackson, I. J. (1989). Climate, water and agriculture in the tropics. Longman Scientific and Technical, Harlow

Kutiel, H. (1987). Rainfall variations in the Galilee (Israel), I. Variations in the spatial distribution in the periods 1931-1960, and 1951-1980. J. Hydrol. 94: 331-344

Kutiel, H. (1988). Rainfall variations in the Galilee (Israel), II Variations in the temporal distribution between 1931-1960, and 1951-1980. J. Hydrol. 99: 179-185

Kutiel, H. (1989). Factors associated with distribution of rainfall in the Galilee, Israel. Pirineos 134: 41-58
Kutiel, H. (1993). L'incertitude du régime pluviométrique en Méditerranée orientale. Publications de l'Association Internationale de Climatologie, Vol. 6, La sécheresse en Méditerranée. Association Internationale de Climatologie. Thessaloniki

Linacre, E. (1991). Climate data and resources. Routledge, London

Newmark, J. (1991). Statistics and probability in modern life. 5 th edn. Saunders College Publishing, Fort Worth

Pielke, R. A., Mehring, P. (1977). Use of mesoscale climatology in mountainous terrain to improve the spatial repre. sentation of mean monthly temperatures. Mon. Weather Rev. 105: 108-112

Rosenberg, M. (1969). Choix d'un modèle régional expliquant la répartition des précipitations annuelles dans l'espace en fonction des facteurs climatiques et topographiques. C. r Acad. Sci. Paris 268: 2761-2764

Russo, J. M., Liebhold, A. M., Kelley, J. G. W. (1993) Mesoscale weather data as input to a gypsy moth (Lepidoptera: Lymantriidae) phyenology model. J. econ. Entomol. 86: 838-844

Sadan, E., Ben-Zvi, R. (1987). The value of institutional change in Israel's water economy. Wat. Resour. Res. 23 $1-8$

Sharon, D. (1972). The spottiness of rainfall in desert area. J. Hydrol. 17: 161-175

Sharon, D. (1979). Local rains in the Arava. Eretz-ha-NegevThe land of the Negev (in Hebrew), p. 119-124

Sharon, D., Kutiel, H. (1986). The distribution of rainfall intensity in lsrael, its regional and seasonal variations and its climatological evaluation. J. Climatol. 6: 277-291

Stanhill, G., Rappaport, C. (1988). Temporal and spatial variations in the volume of rain falling annually in Israel. Israel J. Earth Sci. 37: 211-221

Stephenson, P. M. (1968). Objective assessment of adequate numbers of rain gauges for estimating areal rainfall depths. Proceedings, IASH General Assembly (Bern 1967), Publ. No. 78, Bern, p. 252-264

Striem, H. L. (1967). Rainfall groupings in the Middle East Bull int. Ass. scient. Hydrol. 12: 59-64

Sumner, G. (1988). Precipitation process and analysis. John Wiley \& Sons, Chichester

Wolfson, N. (1975). Topographical effects on standard normals of rainfall over Israel. Weather 30: 138-140

WMO (1965). Guide to hydrometeorological practices. World Meteorological Organization, Geneva

Manuscript first received: March 2, 1994

Revised version accepted: July 14, 1994

Editor: V. Meentemeyer, Athens, Georgia, USA 